\title{
A NOTE ON PERMUTATIONS IN AN ARBITRARY FIELD
}

\section{CARLITZ ${ }^{1}$}

The writer [1] has proved that every permutation on the numbers of the finite field $G F(q)$ is generated by the special permutations

$$
x^{q-2}, \quad \alpha x+\beta \quad(\alpha, \beta \in G F(q), \alpha \neq 0) .
$$

Let $F$ denote an arbitrary field. Define the function

$$
x^{*}= \begin{cases}x^{-1} & (x \in F, x \neq 0), \\ 0 & (x=0) .\end{cases}
$$

Clearly $x^{*}$ defines a permutation of $F$.

The following theorem holds.

THEOREM 1. Every transposition ( $\alpha \beta)$, where $\alpha, \beta \in F$ is finitely generated by the special permutations

$$
x^{*}, \quad \gamma x+\delta \quad(\gamma, \delta \in F, \gamma \neq 0) .
$$

The proof (compare [1]) follows from consideration of the function

$$
g(x)=-\alpha^{2}\left(\left((x-\alpha)^{*}+\frac{1}{\alpha}\right)^{*}-\alpha\right)^{*},
$$

where $\alpha$ is a fixed nonzero number of $F$. It is easily verified that $g(x)$ represents the transposition $(0 \alpha)$.

Let $G=G(F)$ denote the group consisting of all finite products

$$
t_{1} t_{2} \cdots t_{n}
$$

where the $t_{j}$ are arbitrary transpositions $(\alpha \beta)$. As an immediate corollary of Theorem 1 we have

THEOREM 2. The group $G$ is generated by the special permutations (3).

\section{REFERENCE}

1. L. Carlitz, Permutations in a finite field, Proc. Amer. Math. Soc. 4 (1953), 538.

DURE UNIVERSITY

Received by the editors December 15, 1961.

${ }^{1}$ Supported in part by the National Science Foundation grant G 16485. 